\title{
Im Kontalkt: Mediation und Angewandte Gerontologie
}

\section{Hochschule Mannheim erweitert Studienangebot für Berufserfahrene}

\Z Zwei interdisziplinäre und berufsbegleitende Studiengänge der Hochschule Mannheim starten zum Wintersemester. Beide Hochschulweiterbildungen wenden sich an Menschen mit Berufserfahrung, die in Führungsoder Schlüsselfunktionen tätig sind.

Das neue Kontaktstudium Mediation unter der Leitung von Prof. Dr. iur. Ulla Törnig beschäftigt sich mit Prozessen und Strategien verhandlungsund verständigungsorientierter Konfliktbearbeitung. Mit der Mediation eröffnet sich ein Verfahren für verschiedene Arbeitsfelder (z.B. Familien-, Wirtschafts-, Interkulturelle Mediation). Es orientiert sich am Format der „integrierten Mediation“ und ermöglicht es, die beteiligten Parteien zu selbstverantwortlichen Konfliktregelungen und Lösungen zu führen. Das Angebot richtet sich an Führungskräf- te im Human Ressource Management, die im Konfliktmanagement eingebunden sind, aber auch an die in Konflikt- und Streitangelegenheiten tätigen Berufsgruppen aus Recht, Psychologie, Medizin, Sozialer Arbeit, Pädagogik, Quartiersmanagement. Das Studium schließt mit einer Zertifizierung gemäß $\S \S 5$ und 6 MediationsG, ZMediat-AusbV ab und wird in Zusammenarbeit mit der Paritätischen Akademie Süd in Stuttgart durchgeführt. Start ist im November 2017.

Mit den vielfältigen Fragen des Alterns befasst sich der Kontaktstudiengang „Angewandte Gerontologie, Multidisziplinäre Interventionsgerontologie und Gerontopsychiatrie" auf Masterniveau. Die wissenschaftliche Hochschulweiterbildung unter Leitung von Prof. Dr. phil. Astrid HedtkeBecker richtet sich an berufserfahrene
Fachleute aus dem Sozial- und Gesundheitswesen, die Schlüssel- und Leitungsfunktionen innehaben und mit alten Menschen arbeiten. Schwerpunkte des Studiums sind die Allgemeine Gerontologie, die multidisziplinäre Interventionsgerontologie und die Gerontopsychiatrie. Die Studierenden lernen zukünftige Formen der Betreuung, Begleitung und Versorgung von alten sowie gerontopsychiatrisch erkrankten Menschen kennen. Der Studiengang ist Teil des Verbundmasters Zukunft Alter mit Angeboten in Mannheim, Freiburg, München; CASAbschlüsse und ECTS werden gegenseitig anerkannt. Studienbeginn ist im Oktober 2017, Anmeldeschluss ist der 20. August 2017. Der Abschluss lautet Gerontologin/Gerontologe CAS.

www.hs-mannheim.de

\section{Hier steht eine Anzeige.}

\section{并 Springer}

